\title{
Spectrum of Galactic Cosmic Rays Accelerated in Supernova Remnants
}

\author{
Vladimir Ptuskin and Vladimir Zirakashvili \\ Pushkov Institute of Terrestrial Magnetism, Ionosphere and Radio Wave Propagation of the Russian \\ Academy of Science (IZMIRAN), Troitsk, Moscow Region 142190, Russia \\ and \\ Eun-Suk Seo \\ Department of Physics and Institute of Physical Science and Technology, University of Maryland, College \\ Park, MD 20742 USA
}

\begin{abstract}
The spectra of high-energy protons and nuclei accelerated by supernova remnant shocks are calculated taking into account magnetic field amplification and Alfvenic drift both upstream and downstream of the shock for different types of supernova remnants during their evolution. The maximum energy of accelerated particles may reach $5 \cdot 10^{18} \mathrm{eV}$ for Fe ions in Type IIb SNRs. The calculated energy spectrum of cosmic rays after propagation through the Galaxy is in good agreement with the spectrum measured at the Earth.
\end{abstract}

Subject headings: acceleration of particles — shock waves — supernova remnants

\section{Introduction}

Supernova remnants (SNRs) are recognized as the principal sources of Galactic cosmic rays, and the diffusive shock acceleration is accepted as the mechanism of cosmic ray acceleration by a supernova blast wave moving through the turbulent interstellar medium. Accelerated by supernova shocks, the energetic particles diffuse in the interstellar magnetic fields and fill the entire Galaxy. Clear evidence for particle acceleration in SNRs is given by observations of non-thermal radio, X-ray, and gamma-ray radiation. It is experimentally established from H.E.S.S. (High Energy Stereoscopic System) data that there are cosmic-ray particles with energies exceeding $10^{14} \mathrm{eV}$ in the shell of the supernova remnant RX J1713.7-3946 (Aharonian et al. 2007).

For a time, the theoretical estimates of maximum proton energy were at a level of $E_{\max } \approx 10^{13}$ $10^{14} \mathrm{eV}$ (Lagage \& Cesarsky 1983). The work of Bell (2004) demonstrated that a very large ran- dom magnetic field $\delta \mathrm{B} \gg B_{\text {ism }}$ (where $B_{\text {ism }} \approx 5 \mu G$ is the average interstellar field upstream of the shock) can be generated by a cosmic-ray streaming instability in the precursor of strong shocks. It results then in efficient confinement of energetic particles in the shock vicinity, and it may raise the maximum particle energy by about two orders of magnitude (the exact value depends on the supernova parameters, see below). Remarkably, data on synchrotron X-ray emission from a number of young SNRs proved the presence of strong magnetic fields 150 to $500 \mu \mathrm{G}$ (Völk et al. 2005) that can be naturally explained by the effect of cosmicray streaming instability. An excellent concise review on the origin of observed cosmic-ray spectrum was presented by Hillas (2006).

In the present work we calculate the steady state spectrum of cosmic rays in the Galaxy using recent results on magnetic field amplification in SNRs and including different types of SNRs in the consideration. A numerical code is employed in the simulations of particle acceleration and the 
SNR shock evolution.

\section{Modelling of cosmic ray acceleration in supernova remnants}

Because of high efficiency of shock acceleration, the spectrum of cosmic rays should be selfconsistently determined with the account of shock modification caused by the pressure of accelerated particles. We are studing cosmic ray acceleration and the evolution of a supernova blast wave with the use of our numerical code described in (Zirakashvili \& Ptuskin 2009a,b). The hydrodynamic equations are solved together with the diffusion-convection transport equation for the cosmic ray distribution function $f(t, r, p)$, which depends on time $t$, radial distance from the point of supernova explosion $r$ (here the spherical symmetry is assumed), and the particle momentum $p$. The full system of equations is the following:

$$
\begin{gathered}
\frac{\partial \rho}{\partial t}=-\frac{1}{r^{2}} \frac{\partial}{\partial r} r^{2} u \rho \\
\frac{\partial u}{\partial t}=-u \frac{\partial u}{\partial r}-\frac{1}{\rho}\left(\frac{\partial P_{g}}{\partial r}+\frac{\partial P_{c}}{\partial r}\right), \\
\frac{\partial P_{g}}{\partial t}=-u \frac{\partial P_{g}}{\partial r}-\frac{\gamma_{g} P_{g}}{r^{2}} \frac{\partial r^{2} u}{\partial r}-\left(\gamma_{g}-1\right)(w-u) \frac{\partial P_{c}}{\partial r}, \\
\frac{\partial f}{\partial t}=\frac{1}{r^{2}} \frac{\partial}{\partial r} r^{2} D(p, r, t) \frac{\partial f}{\partial r}-w \frac{\partial f}{\partial r}+\frac{\partial f}{\partial p} \frac{p}{3 r^{2}} \frac{\partial r^{2} w}{\partial r} \\
+\frac{\eta \delta\left(p-p_{i n j}\right)}{4 \pi p_{i n j}^{2} m} \rho(R-0, t)(u(R-0, t)-\dot{R}) \delta(r-R(t)) .
\end{gathered}
$$

Here $\rho$ is the gas density, $u$ is the gas velocity, $P_{g}$ is the gas pressure, $P_{c}=4 \pi \int p^{2} d p v p f / 3$ is the cosmic ray pressure, $w(r, t)$ is the advective velocity of cosmic rays, $\gamma_{g}$ is the adiabatic index of the gas, and $D(r, t, p)$ is the cosmic ray diffusion coefficient. It is assumed that the diffusive streaming of cosmic rays results in the generation of magnetohydrodynamic waves and provides the Bohm diffusion coefficient $D_{B}=v p c /(3 Z e B)$ for accelerating particles of charge $Z e$ and velocity $v(c$ is the speed of light).

The essential feature of our calculations is the inclusion of the Alfvenic drift effect for particle transport. The Alfven velocity $V_{A}=B / \sqrt{4 \pi \rho}$ is not negligible in comparison to the gas velocity $u$ downstream of the shock if the magnetic field is significantly amplified as indicated by the observations of the synchrotron $\mathrm{X}$-ray radiation. The effective scattering of energetic particles near the shock is provided by Alfven waves generated by a resonant cosmic ray streaming instability. Unstable Alfven waves propagate in the direction opposite to the cosmic ray gradient. We also assume that the growth of waves is balanced by a nonlinear damping that is strong enough for high wave amplitudes. The energy which cosmic rays lose due to the wave generation eventually goes into the gas heating (see the last term in Eq. (3)). The cosmic ray gradient is negative upstream of the shock and the waves generated propagate in the positive direction. The waves transmitted downstream of the shock are damped and regenerated by the cosmic ray gradient in this region. The cosmic ray gradient is positive here because of the adiabatic losses of cosmic ray particles (see numerical calculations of Zirakashvili \& Ptuskin (2009a)). As a result the generated Alfven waves propagate in the negative direction downstream of the shock.

The situation is somewhat different for the highest energy particles. An electric current of these particles amplifies the magnetic field upstream of the shock via a non-resonant streaming instability Bell (2004). The generated random magnetic fields are not Alfven waves but almost purely growing magnetic disturbances. This amplified field plays a role of the regular field for lowenergy particles. They produce Alfven waves that propagate along the tangled magnetic field lines. The amplified magnetic field is almost isotropic even downstream of the shock in spite of compression at the shock front. This is because the shock front is corrugated by density fluctuations generated by the streaming instability upstream of the shock (Zirakashvili \& Ptuskin 2008). So the transport velocity for highest energy particles is close to the plasma velocity $u$ in contrast to low-energy particles. For the sake of simplicity we neglect this below and use the Alfven transport for all energies.

Taking effects mentioned above into account we set the cosmic ray advection velocity equal to $w=u-V_{A} / \sqrt{3}$ downstream of the shock. Because of this the accelerating particles "feel" a smaller compression ratio and acquire a softer energy spec- 
trum compared to the usual assumption $w=u$. We set $w=u+V_{A} / \sqrt{3}$ upstream of the shock to account for the Alfvenic drift effect there. The damping of Alfven waves created by the cosmic ray streaming instability results in a very important effect of gas heating upstream of the shock described by the last term in Eq. (3). This effect limits the total shock compression ratio.

The spatial dependence of amplified magnetic field is taken in the form $B(r)=\sqrt{4 \pi \rho_{0}} \frac{\dot{R} \rho}{M_{A} \rho_{0}}$ where $\rho_{0}$ is the gas density of the circumstellar medium and $M_{A}$ is some constant. We employ results of Völk et al. (2005) in the analysis of Xray radiation from young SNRs and assume that magnetic energy density $B^{2} / 8 \pi$ downstream of the shock is $3.5 \%$ of the ram pressure $\rho u_{\mathrm{sh}}^{2}$ that determines the constant $M_{A}=23$. It is worth noting that this relation is in good agreement with the modelling of cosmic ray streaming instability in young SNRs (Zirakashvili \& Ptuskin 2008).

The last term in Eq. (4) corresponds to the injection of thermal protons with momenta $p=p_{i n j}$ and mass $m$ at the shock at $r=R(t)$. The dimensionless parameter $\eta$ determines the injection efficiency. The injection efficiency of thermal ions in the process of shock acceleration $\eta=0.1 u_{\mathrm{sh}} / c$ is taken in accordance with the paper Zirakashvili (2007); here $u_{\mathrm{sh}}(t)$ is the time-varying shock velocity.

The maximum particle momentum $p_{\max }$ reached in a process of diffusive shock acceleration can be roughly estimated from the condition $D_{B}\left(p_{\max }\right) \sim$ $0.1 u_{\mathrm{sh}} R_{\mathrm{sh}}$ with $D_{B}$ calculated for the upstream magnetic field, which is about 5 times smaller than the downstream field. This gives an order of magnitude estimate $p_{\max } c / Z \sim 20\left(u_{\mathrm{sh}} / 10^{3} \mathrm{~km} \mathrm{~s}^{-1}\right)^{2} R_{\mathrm{sh}} \sqrt{n}$ $\mathrm{TeV}$, where the shock radius is $R_{\mathrm{sh}} \mathrm{pc}$, and the interstellar gas number density $n \mathrm{~cm}^{-3}$.

It can be shown, see e.g. Ptuskin \& Zirakashvili (2005), that the transformation of supernova explosion energy to cosmic rays becomes efficient from the beginning of the Sedov stage of the shock evolution (i.e. when the mass of supernova ejecta becomes equal to the mass of swept-up gas) and continues later on. As a result, the characteristic knee arises in the overall spectrum of particles accelerated by the evolving supernova remnant. The position of knee $p_{\text {knee }}$ can be estimated from the above equations for $p_{\max }$ where $u_{\text {sh }}$ and $R_{\text {sh }}$ are determined at the time when the Sedov stage begins. It gives approximately

$$
p_{\text {knee }} c / Z \sim 1 \cdot 10^{15} E_{51} n^{1 / 6} M_{\mathrm{ej}}^{-2 / 3} \mathrm{eV} .
$$

Here $E_{51}$ is the kinetic energy of the supernova explosion in units of $10^{51} \mathrm{erg}$ and $M_{\mathrm{ej}}$ is the mass of supernova ejecta measured in solar masses.

If the presupernova had a dense star wind with velocity $u_{w}$ and the mass loss rate $\dot{M}$ before the explosion, the shock may enter the Sedov stage while propagating through the wind material with mass density $\rho_{w}=\dot{M} /\left(4 \pi u_{w} r^{2}\right)$. Eq. (5) should be replaced in this case by the following equation:

$$
p_{\text {knee }} c / Z \sim 8 \cdot 10^{15} E_{51} \sqrt{\dot{M}_{-5} / u_{w, 6}} M_{\mathrm{ej}}^{-1} \mathrm{eV}
$$

We have performed numerical simulations of cosmic ray acceleration for 4 types of supernova remnants (they constitute about 90 percents of all supernovae).

1. Type Ia SNRs with the following parameters: kinetic energy of explosion $E=10^{51} \mathrm{erg}$, number density of the surrounding interstellar gas $n=0.1 \mathrm{~cm}^{-3}$, and mass of ejecta $M_{\mathrm{ej}}=1.4 M_{\odot}$. Also important for accurate calculations is the index $k$, which describes the power law density profile $\rho_{s} \propto r^{-k}$ of the outer part of the star that freely expands after supernova explosions; $k=7$ for Type Ia supernova.

2. Type IIP SNRs with parameters $E=$ $10^{51} \mathrm{erg}, n=0.1 \mathrm{~cm}^{-3}, M_{\mathrm{ej}}=8 M_{\odot}$, and $k=12$.

3. Type Ib/c SNRs with $E=10^{51}$ erg exploding into the low density bubble with density $n=0.01$ $\mathrm{cm}^{-3}$ formed by a progenitor star that starts off as an O star, goes through a RSG (Red Super Giant) phase, and ends its life as a Wolf-Rayet star, see e.g. Dwarkadas (2007). The ejecta mass is $M_{\mathrm{ej}}=2 M_{\odot}$ and $k=7$.

4. Type IIb SNRs with $E=3 \cdot 10^{51} \mathrm{erg}, n=$ $0.01 \mathrm{~cm}^{-3}$ and $M_{\mathrm{ej}}=1 M_{\odot}$. Before entering the rarefied bubble, the blast wave goes through the dense wind emitted by the progenitor star during its final RSG stage of evolution. We assume that the mass loss rate by the wind is $\dot{M}=10^{-4} M_{\odot} /$ yr and the outer wind radius is $5 \mathrm{pc}$.

A discussion about properties of SNRs produced by core collapse supernovae can be found in Chevalier (2005). 
We accept the following relative rates for the four types of supernovae described above: $0.32,0.44,0.22$ and 0.02 respectively. The first three rates are taken from the work of Smartt et al. (2009) based on the statistics of supernovae within $28 \mathrm{Mpc}$ of the Galaxy (they also agree with the Galactic supernova rates derived by Leaman (2008)). The statistics for rare Type IIb events is not sufficiently reliable. These supernovae determine the cosmic ray intensity at energies above about $3 \times 10^{17} \mathrm{eV}$ and our rate 0.02 is chosen to fit the data on these ultra-high energy cosmic rays. According to Smartt et al. (2009), the Type IIb supernovae rate can be as high as 0.04. Notice also that the fluctuation effect is very strong for these rare events.

The calculated cosmic ray spectra produced over the lifetime of each type of supernovae are shown in Figure 1 under the assumption that only protons are accelerated. Here $Q(p)=4 \pi p^{2} F(p)$, where $F(p)$ is the distribution of all accelerated particles injected in the interstellar medium over the SNR lifetime. The total number of accelerated particles is $\int Q(p) d p$. It was assumed that the acceleration ceased at $t_{c}=10^{5} \mathrm{yr}$. The shock velocity at this moment is close to $200 \mathrm{~km} / \mathrm{s}$, and the maximum energy of protons confined in the supernova remnant is $\sim 5 \mathrm{TeV}$. All particles with higher energies accelerated earlier have left the remnant. The maximum particle energy at late stages of shock evolution can be two-three orders of magnitude smaller if one takes into account the possible damping of turbulence in the shock precursor due to ion-neutral collisions or non-linear wave interactions (Ptuskin \& Zirakashvili 2003).

The function $F(p)$ was calculated as the sum of two integrals: the integral taken at $t_{c}$ over the volume of supernova remnant $(4 \pi) \int_{0}^{R_{\mathrm{sh}}\left(t_{c}\right)} f\left(t_{c}, r, p\right) r^{2} d r$, and the integral over time of the diffusion flux of accelerated particles through the boundary of the calculation domain $\left.\left[4 \pi r^{2} \int_{0}^{t_{c}}(-D \partial f / \partial r) d t\right]\right|_{r_{b}}$. The source function $Q(p)$ should be multiplied by $\nu_{\mathrm{sn}}$, where $\nu_{\mathrm{sn}}$ is the supernova rate per unit volume to obtain the density of cosmic ray sources. Figure 1 shows that about $1 / 3$ of supernova explosion kinetic energy $E$ goes to cosmic rays, which is in agreement with the empirical model of cosmic ray origin.

The spectrum of accelerated energetic ions other than protons has the same shape if expressed

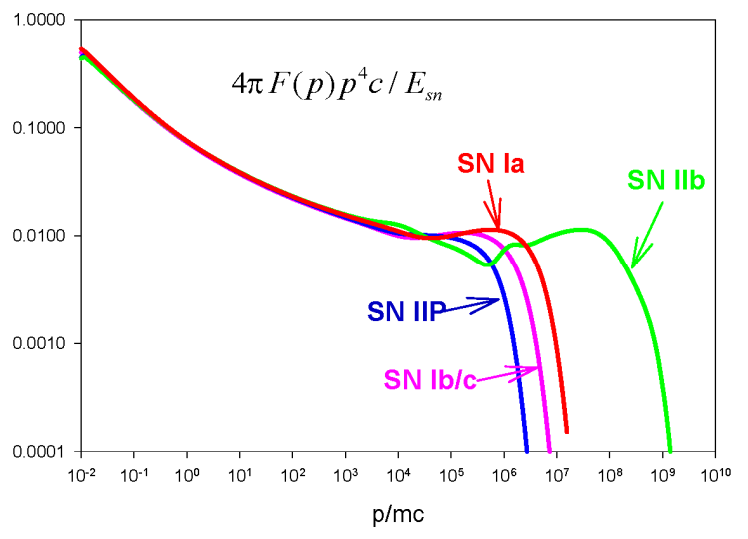

Fig. 1. - Source spectra produced by supernovae Type Ia (solid line), Type IIP (dash line), Type $\mathrm{Ib} / \mathrm{c}$ (dotted line), and Type IIb (dash-dot line) assuming that the accelerated particles are protons.

as a function of magnetic rigidity $Q(p / Z)$ with the appropriate absolute normalization determined by the injection process at thermal energies.

The relativistic ions released into interstellar space from numerous supernova remnants, diffuse in galactic magnetic fields, interact with interstellar gas, and finally escape through the cosmic ray halo boundaries into intergalactic space, where the density of cosmic rays is negligible. The main characteristics of cosmic ray propagation in the Galaxy needed for calculation of the cosmic ray spectrum in the so called leaky-box approximation is the escape length $X_{e}$, that is the average matter thickness traversed by cosmic rays before they exit from the Galaxy, see e.g. Strong et al. (2007). The cosmic ray intensity obeys the relation $I \propto \nu_{\mathrm{sn}} Q\left(X_{e}^{-1}+\sigma / m_{a}\right)^{-1}$, where $\sigma$ is the nuclear spallation cross section for a given type of relativistic nuclei moving through the interstellar gas, $m_{a}$ is the mean interstellar atom mass.

The value of the escape length is determined from the relative abundance of secondary nuclei (primarily from the Boron-to-Carbon ratio) in cosmic rays. Based on the paper Jones et al. (2001), we choose the escape length in the form

$$
X_{e}=11.8(v / c)(p / 4.9 Z \mathrm{GV})^{-0.54} \mathrm{~g} / \mathrm{cm}^{2}
$$

at $p / Z \geq 4.9 \mathrm{GV} ;$ and $X_{e} \propto v / c$ at $p / Z<4.9$ GV. Eq. (7) means that the resulting spectrum is 
steeper than the source spectrum by 0.54 at high enough energies. We use Eq. (7) at all energies despite the fact that the $\mathrm{B} / \mathrm{C}$ ratio is not measured above $100-1000 \mathrm{GeV}$.

The source normalization for nuclei from protons to Iron was made in our calculations from the fit to observed cosmic ray composition at one reference energy $10^{3} \mathrm{GeV}$.

The results of the calculations are shown in Figure 2a for the interstellar spectra of protons, Helium and Iron nuclei at kinetic energies per nucleon $1 \mathrm{GeV} / \mathrm{n}<E<10^{3} \mathrm{GeV} / \mathrm{n}$ where the charge resolution of cosmic ray experiments is high and the escape length $X_{e}(E)$ is known. The agreement between our theoretical predictions and the observations of cosmic-ray energy spectra supports the validity of our acceleration and propagation models. (The discordance between the calculated interstellar and the measured at the Earth spectra at energies below $10 \mathrm{GeV} / \mathrm{n}$ is due to the solar wind modulation effect.)

The combined spectrum of all protons and ions with particle energies $E \geq 10^{3} \mathrm{GeV}$ is shown by the solid line in Figure 2b. It was assumed that the charge composition of accelerated particles was the same in all types of SNRs except that the highest-energy part of the spectrum produced by Type Ib/c supernovae at $\mathrm{pc} / Z>10^{5} \mathrm{GeV}$ had no hydrogen. This reflects the composition of presupernova, the Wolf-Rayet star with a fast $\mathrm{H}$-poor wind. It was assumed that the shock produced by a supernova explosion first propagates through the 5 solar masses of the Wolf-Rayet star material uniformly spread in the central part of the wind bubble and propagates through the material with normal composition after that. It may explain why the protons do not dominate in the cosmic-ray composition at the knee Antoni et al. 2005; Budnev et al. 2007). In fact the proton-toHelium ratio in cosmic rays is probably decreasing with energy starting at smaller energies $10^{2}-10^{3}$ $\mathrm{GeV} /$ nucleon (Seo 2007). We plan detailed investigation of this point in a separate paper.

The calculated spectra show remarkably good overall fit to observations up to energies $\sim 5 \cdot 10^{9}$ $\mathrm{GeV}$, where the transition to extragalactic cosmic rays with the characteristic GZK suppression above $3 \times 10^{10} \mathrm{GeV}$ likely occurs. To a good approximation the bending of the observed spectrum at around the knee energy $3 \cdot 10^{6} \mathrm{GeV}$ is repro-
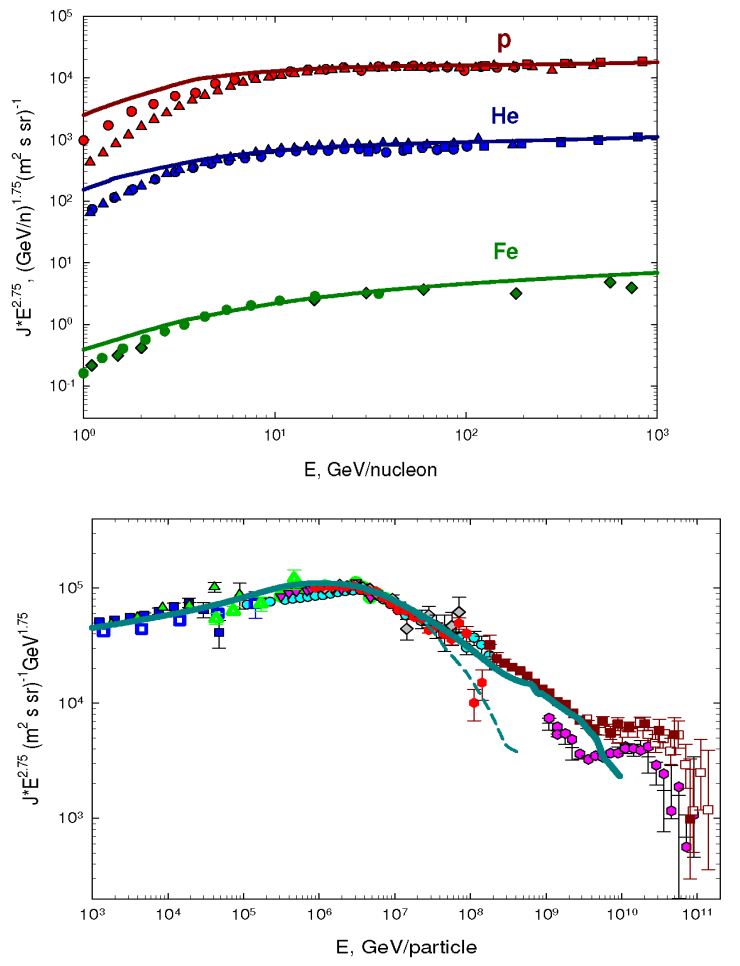

Fig. 2.- (a) The calculated interstellar (not corrected for solar modulation at low energies) spectra of protons, Helium, and Iron below energy $10^{3} \mathrm{GeV} / \mathrm{n}$. Observational data from AMS (Alcaraz et al. 2000), ATIC2 (Panov et al. 2006), BESS-TeV (Haino et al. 2004), HEAO-3 (Engelmann et al. 1990), and TRACER (Ave et al. 2008) experiments are shown. (b) The all particle spectrum above $10^{3}$ $\mathrm{GeV}$ calculated under the assumptions that escape length is determined by Eq. (7) at all energies (solid line) and has a cutoff at $2 \cdot 10^{7} Z \mathrm{GeV}$ (dash line). See Blumer et al. (2009) for references to the observational data shown by grey symbols. 


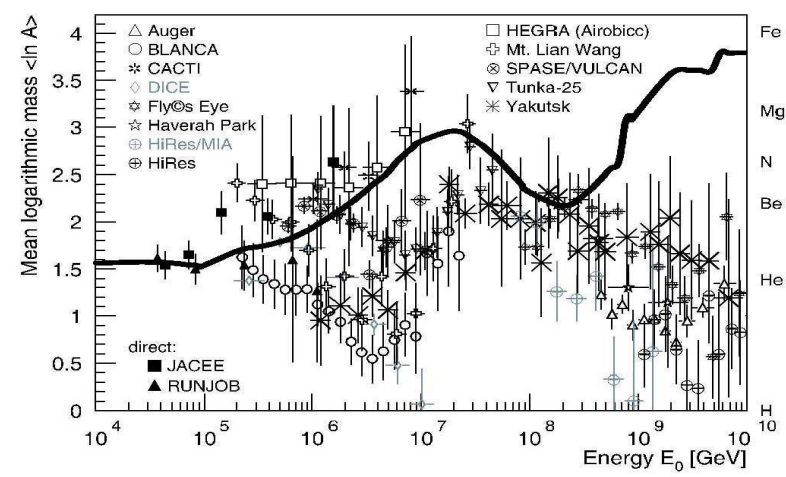

Fig. 3.- Calculated mean logarithmic mass of produced in the Galaxy cosmic rays (thick solid line) compared to observational data based on the average depth of shower maximum as presented in Blumer et al. (2009).

duced although no special efforts were made to force the theory to fit the data. The bending is due to the combined effect of the summation over different types of SNRs and over different types of accelerated nuclei.

The complicated chemical composition of high energy cosmic rays is illustrated in Figure 3 where the calculated mean logarithmic atomic number of cosmic rays $<\ln (A)>$ is presented. The increase of $<\ln (A)>$ at energies from $10^{5} \mathrm{GeV}$ to $10^{7} \mathrm{GeV}$ is due to the dependence of the knee position on charge $p_{\text {knee }} \propto Z$ for each kind of ion accelerated in Types Ia, IIP, Ib/c SNRs. Type IIb SNRs with normal composition dominate at rigidities $p / Z>5 \cdot 10^{6} \mathrm{GV}$. They have a knee at about $p_{\text {knee }} / Z \approx 5 \cdot 10^{7} \mathrm{GV}$ and provide progressively heavier composition to the very high energies. It should be pointed out that the increase of $<\ln (A)>$ at $E>3 \times 10^{8} \mathrm{GeV}$ predicted in our calculations is not supported by the available observations. If confirmed, these observations may signify the dominant contribution of extragalactic cosmic rays with light composition at these energies.

The obtained cosmic ray spectrum shown by the solid line in Figure $2 \mathrm{~b}$ is very attractive for the explanation of cosmic ray data. However, the use of the escape length (7) at ultra high energies is not justified. Experimentally, the value of $X_{e}$ is determined from the abundance of secondary nuclei in cosmic rays with good statistics only up to about $100 \mathrm{GeV} / \mathrm{n}$ (Strong et al. 2007). If cosmic ray transport in the Galaxy is described as diffusion, the diffusion coefficient can be expressed through the escape length as $D \approx v \mu H / 2 X_{e}$ (here $\mu \approx 0.003 \mathrm{~g} / \mathrm{cm}^{2}$ is the surface mass density of Galactic gas disk, $H \approx 4 \mathrm{kpc}$ is the height of the Galactic cosmic-ray halo), which gives $D \approx$ $1.3 \cdot 10^{28}(p c / Z \mathrm{GeV})^{0.54} \mathrm{~cm}^{2} / \mathrm{s}$. The diffusion approximation can be used when the diffusion mean free path $3 D / v$ is less than the size of the system $H$, which results in the condition $p c / Z<2 \cdot 10^{7}$ $\mathrm{GeV}$. At somewhat higher energies the particles accelerated in the galactic disk fly straight away from the Galaxy with a flat (source) energy spectrum and close to hundred percent anisotropy. Certainly this picture does not represent the reality that may be due to the strong intermittency of very high energy cosmic rays produced by random short bursts of not very numerous sources. The dash line in Figure $2 \mathrm{~b}$ shows the results of calculations made under the assumption that cosmic ray particles with energies $p c>2 \cdot 10^{7} Z \mathrm{GeV}$ freely escape from the Galaxy without being detected by observer at the Earth. The predicted spectrum may fit observations only below about $5 \cdot 10^{7} \mathrm{GeV}$ in this case.

The validity of diffusion approximation extends to higher energies in the diffusion model with distributed reacceleration on the interstellar turbulence where $X_{e} \propto(p / Z)^{-1 / 3}$ at high rigidities, see Seo \& Ptuskin (1994). However, this scaling does not reproduce the observed cosmic ray spectrum for the calculated source spectrum, see also discussion in Section 3. It is worth noting that the uncertainty in our knowledge of parameters of the interstellar turbulence does not allow to decide between two basic models of cosmic ray propagation: the plain diffusion model and the diffusion model with distributed reacceleration, see Ptuskin et al. (2006b).

The physical pattern of cosmic ray propagation is different in the models with a Galactic wind. The wind model with selfconsistently calculated cosmic-ray transport coefficients reproduces well the data on secondary nuclei (Ptuskin et al. 1997). The supersonic wind is probably terminated by the shock at $\sim 0.5 \mathrm{Mpc}$ from the Galactic disk. The confinement of very high energy cosmic rays in the Galaxy can be more efficient in this model as compared to the diffusion model with a static flat 
halo of the size $\sim 4 \mathrm{kpc}$ discussed above. In any case, trajectory calculations in galactic magnetic fields are needed to study cosmic ray propagation when the diffusion approximation breaks up at ultra high energies. The detailed consideration of this issue is beyond the scopes of the present paper.

\section{Discussion and Conclusion}

We have calculated the steady state spectrum of cosmic rays produced by SNRs in the Galaxy. Our new numerical code (Zirakashvili \& Ptuskin 2009a b) for modelling of particle acceleration by spherical shocks with the back reaction of cosmic ray pressure on the shock structure was used in the calculations. The significant magnetic field amplification in young SNRs inferred from the observations of their synchrotron X-ray radiations (Völk et al. 2005), and most probably produced by cosmic ray streaming instability, was also introduced in the calculations. It led to the inclusion of the Alfvenic drift in the equation for particle transport downstream of the shock. Four different types of SNRs with relative burst rates taken from Smartt et al. (2009) were included in the calculations. The escape length Eq.(7) from the work of Jones et al. (2001) was used to describe the propagation of cosmic rays in the Galaxy in the leakybox approximation. The normalization to the observed intensity and chemical composition of cosmic rays was made at $10^{3} \mathrm{GeV}$ energy.

The results are illustrated by the solid lines in Figures 2 when Eq. (7) for $X_{e}$ is used without limitation and by the dash line when it is limited by the applicability of the diffusion approximation for cosmic ray propagation in the diffusion model with a flat static halo. The solid lines reproduce well the entire cosmic ray spectrum up to $\sim 3 \cdot 10^{9} \mathrm{GeV}$ while the dash line makes it up to less than $\sim 5 \cdot 10^{7}$ $\mathrm{GeV}$. Further investigations of cosmic ray propagation in galactic magnetic fields at ultra high energies are needed to refine the predicted shape of the spectrum produced by the Galactic SNRs at ultra-high energies. This is important in light of the discussion about transition from the Galactic to extragalactic component in the observed cosmic ray spectrum Berezinsky et al. 2006; Hillas 2006).

Our results can be compared to the earlier work
Berezhko \& Völk (2007), where the Alfvenic drift effect was not taken into account and only Type Ia SNRs that represents about $30 \%$ of all SNRs and can not efficiently accelerate particles to ultrahigh energies were considered. The absence of Alfvenic drift led to a very flat cosmic ray source spectrum, which required too strong dependence of the escape length on rigidity $X_{e} \propto(p / Z)^{-0.75}$ inconsistent with the data on secondary nuclei. In fact, this problem with a too hard predicted source spectrum was the main motivation for the present work. We included the effect of Alfvenic drift downstream of the shock and found that the resulting source spectrum fits the observations. To show the importance of this effect clearly, we accepted basically the same set of other parameters and assumptions of the model of shock acceleration in SNRs as Berezhko \& Völk (2007). Also, three additional types of SNRs were included into the consideration.

The present work demonstrated that supernovae can in principle produce the source spectrum of galactic cosmic rays required by the empirical model of cosmic ray origin. More work is needed to understand how robust are our results.

First of all it is more detailed analysis of MHD effects that accompany the process of the diffusive shock acceleration in evolving SNRs. In particular, a further investigation of generation and transport of MHD turbulence at astrophysical shocks is necessary. For simplicity we described the properties of MHD turbulence in our calculations by only one parameter $M_{A}$. Its representative value $M_{A}=23$ corresponding to the typical magnitude of amplified magnetic field determined by Völk et al. (2005) from the observations of non-thermal X-ray emission from young SNRs gives the particle spectrum that is in accordance with cosmic-ray observations. However we checked that this result strongly depends on the assumed value of $M_{A}$ which regulates the spectral slope via Alfvenic drift downstream of the shock. As an example, for $M_{A}=15$ the spectrum of accelerated particles is so steep that the escape length $X_{e} \propto(p / Z)^{-1 / 3}$ typical for the propagation model with distributed reacceleration would reproduce the observed cosmic ray spectrum. The problem is however that the streaming instability of particles with steep spectrum can hardly generate the strong magnetic field at large scales that 
is needded for an acceleration of high-energy particles. All this sets one thinking about possible self-consistent mechanism that maintains the specific values of $M_{A}$.

The Alfvenic drift effects downstream of the shock will be verified in the nearest future via combined observations of young supernova remnants in Fermi, HESS, MAGIC, VERITAS and some other experiments. The observations of older remnants will also help to understand how the maximum energy of accelerated particles depends on the age of a remnant. Although the maximum energy probably quickly decreases in the old remnants (see Ptuskin \& Zirakashvili (2003)), we neglect this effect in our present calculations since it would require an introduction of an additional rather uncertain parameter.

A further work is also necessary for determination of the dependence of injection efficiency $\eta$ on the shock parameters. The injection efficiency has strong influence on the resulting particle spectra. It is because the presence of the Alfvenic drift downstream of the shock steepens the spectrum of accelerated particles. So, it is more difficult to modify the shock by the pressure of high energy particles compared to the case without the Alfvenic drift.

The assumed strong heating in the shock precursor also influences the resulting particle spectrum. Although the main part of the cosmic ray energy that goes to the Alfven wave generation is eventually transformed into the gas heating, the level of Alfvenic turbulence $(\delta B / B \sim 1)$ is high enough to provide the scattering of cosmic ray particles in the regime close to the Bohm diffusion. Note that quasi-linear estimates of the Alfven wave amplitude give $\delta B / B>>1$ for young supernova remnants (McKenzie \& Völk 1982). In reality Alfven waves generated by the resonant streaming instability probably stops when $\delta B / B$ reaches the value close to 1 because of collisions of the moving adjacent plasma elements similar to results of (Zirakashvili et al. 2008). The gas heating is then provided by weak shocks produced in these collisions.

Our assumption that the composition of accelerated particles is the same for all types of nuclei (except the very high energy part of the spectrum produced by Type Ib/c SNRs) and our ignoring the dispersion of SNR parameters within the same type of supernovae are probably too simplified to correspond to reality. More comprehensive analysis taking into account earlier work (Silberberg et al. 1991; Sveshnikova 2003; Popescu 2007) is needed, although it requires the introduction of additional not well-known astrophysical parameters.

Another difficult problem is accounting for the fluctuations resulting from the discrete nature of supernovae in space and time. Fluctuations of cosmic ray intensity and anisotropy were addressed in particular by Erlykin \& Wolfendale (2006); Ptuskin et al. (2006a).

The authors are grateful to referee Michael Hillas for valuable comments on the manuscript. This work was supported by the Russian Foundation for Basic Research grant 10-02-00110a. The work was partly fulfilled during VSP visit to the University of Maryland, USA where it was supported by the NASA Astronomy and Physics Research and Analysis grant NNX09AC149.

\section{REFERENCES}

Aharonian, F. et al. 2007, A\&A, 464, 253

Alcaraz, J. et al. 2000, Phys. Let. B, 490, 27; 494, 93

Antoni, T. et al. (KASCADE Collab.) 2005, Astropart. Phys., 24, 1

Ave, M. et al. 2008, ApJ, 678, 262

Bell, A.R. 2004, MNRAS, 353, 550

Berezhko, E.G. \& Völk, H.J. 2007, ApJ, 661, L175

Berezinsky, V., Gazizov, A. \& Grigorieva, S. 2006, Phys. Rev. D, 74, 043005

Bluemer, J., Engel, R. \& Hörandel, J.R. 2009, Progress Part. Nuclear Phys., 63, 293

Budnev, N.M. et al. (TUNKA Collab.) Bull. Russian Acad. Sci.: Physics, 71, 474

Chevalier, R. 2005, ApJ, 619, 839

Dwarkadas, V.V. 2007, ApJ, 667, 226

Engelmann, J. et al. 1990, A\&A, 464, 253 
Erlykin, A.D. \& Wolfendale, A.W. 2006, Astropart. Phys., 25, 183

Haino, S. et al. 2004, Phys. Let. B, 594, 35

Hillas, A.M. 2006, J. Phys: Conf. Ser., 47, 168

Jones, F.C., Lukasiak, A., Ptuskin, V. \& Webber, W. 2001, ApJ, 547, 264

Lagage, P.O. \& Cesarsky, C.J. 1983, A\&A, 125, 249

Leaman, J.F. 2008, The Supernova Rate in the Local Universe, $\mathrm{PhD}$ thesis, University of California, Berkeley

McKenzie, J.F., \& Völk, H.J., 1982, A\&A, 116, 191

Panov, A.D. et al. 2006, arXiv:0612.377

Popescu, A.S. 2007, arXiv:0704.2718v1

Ptuskin, V.S., Jones, F.C., Seo, E.S. \& Sina, R.S. 2006, Adv. Space Res., 37, 1909

Ptuskin, V.S., Moskalenko, I.V., Jones, F.C., Strong, A.W. \& Zirakashvili, V.N. 2006, ApJ, 642,892

Ptuskin, V.S., Völk, H.J., Zirakashvili, V.N. \& Breitschwerdt, D. 1997, A\&A, 321, 434

Ptuskin, V.S. \& Zirakashvili, V.N. 2003, A\&A, 403,1

Ptuskin, V.S. \& Zirakashvili, V.N. 2005, A\&A, 429, 755

Seo, E.S. 2007, in Cosmic-Rays and High Energy Universe, ed. T. Shibata, N. Sakaki, Universal Academy Press, Inc. - Tokyo, Japan, p. 19

Seo, E.S. \& Ptuskin, V.S. 1994, ApJ, 431, 705

Silberberg, R., Tsao, C.H., Shapiro, M.M. \& Biermann, P.L. 1990, ApJ, 363, 265

Smartt, S.J, Eldridge, J.J., Crockett, R.M. \& Maund, J.R. 2009, MNRAS, 395, 1409

Strong, A.W., Moskalenko, I.V. \& Ptuskin, V.S. 2007, Annu. Rev. Nucl. Part. Sci., 57, 285

Sveshnikova, L.G. 2003, A\&A, 409, 799
Völk, H.J., Berezhko, E.G. \& Ksenofontov, L.T. 2005 A\&A, 433, 229

Zirakashvili, V.N. 2007, A\&A, 466, 1

Zirakashvili, V.N. \& Ptuskin, V.S. 2008, ApJ, 678, 939

Zirakashvili, V.N. \& Ptuskin, V.S. 2009, in Highenergy gamma-ray astronomy, eds. F.A. Aharonian et al., Melvill, NY, 2009, AIP Conf. Proc. 1085 , p. 336

Zirakashvili, V.N. \& Ptuskin, V.S. 2009, 31st Int. Cosmic Ray Conf., Lodz (Poland), paper HE.1.1

Zirakashvili, V.N., Ptuskin, V.S., \& Volk, H.J., 2008, ApJ, 678, 256

This 2-column preprint was prepared with the AAS LATEX macros v5.2. 